\title{
Nonlinear modelling and optimal control via Takagi-Sugeno fuzzy techniques: A quadrotor stabilization
}

\author{
Miroslav Pokorný1 ${ }^{1}$, Tomáš Dočekal ${ }^{1}$, Danica Rosinová ${ }^{2 *}$
}

\begin{abstract}
Using the principles of Takagi-Sugeno fuzzy modelling allows the integration of flexible fuzzy approaches and rigorous mathematical tools of linear system theory into one common framework. The rule-based T-S fuzzy model splits a nonlinear system into several linear subsystems. Parallel Distributed Compensation (PDC) controller synthesis uses these T-S fuzzy model rules. The resulting fuzzy controller is nonlinear, based on fuzzy aggregation of state controllers of individual linear subsystems. The system is optimized by the linear quadratic control (LQC) method, its stability is analysed using the Lyapunov method. Stability conditions are guaranteed by a system of linear matrix inequalities (LMIs) formulated and solved for the closed loop system with the proposed PDC controller. The additional GA optimization procedure is introduced, and a new type of its fitness function is proposed to improve the closed-loop system performance.

K e y w o r d s: T-S model, PDC control, LMI approach, LQR optimization, GA method, quadrotor
\end{abstract}

\section{Introduction}

Fuzzy logic is an effective tool for modern modelling and controller synthesis since it integrates both numerical data and non-numerical language information [1]. An important feature of fuzzy logic systems as universal approximators is their ability to effectively model multidimensional nonlinear functions [2]. Practical applications have confirmed the quality of standard fuzzy systems that use triangular membership functions, product conjunction, product implication and centre of gravity defuzzification functions [3].

Takagi and Sugeno proposed a fuzzy logic method of modelling nonlinear systems by decomposing them into a set of linear subsystems [4]. Such decomposition enables to express local dynamics in different regions of state space using linear models. The same fuzzy structure is used for driving applications [5]. A widely used approach of nonlinear systems control using Takagi-Sugeno (T-S) fuzzy models is the Parallel Distributed Compensation (PDC) method [6]. The principle of a PDC controller is to derive a linear control law from the corresponding rules of the T-S model to compensate it. The resulting nonlinear state fuzzy controller is a fuzzy aggregation of local linear controllers of fuzzy subspaces. Stability of such fuzzy systems can be analysed by Lyapunov method and Linear Matrix Inequalities (LMIs) which can be efficiently solved using convex optimization techniques [7]. Tanaka and Sugeno have shown that the stability of the T-S fuzzy system can be proved by the existence of a positive definite matrix $\mathrm{P}$ common to all subsystems, satisfying a set of Lyapunov inequalities. Thus, the quadratic stability problem can be considered as a LMIs feasibility problem. The Lyapunov stability method does not directly address the closed-loop performance problem. The desired transient performance, $i e$ rise time and settling time, can be guaranteed by locating the system poles in a specified region. Therefore, the requirement of Lyapunov quadratic stability can be additionally extended by the requirement for the position of all closed-loop system poles in a prescribed circle region. This requirement can be defined in the term of LMIs and the problem of the required pole position can be converted to the problem of LMIs feasibility [8]. Nevertheless, such a circle region guarantees only a lower bound of the decay rate and the damping ratio.

In the case described above, the closed-loop performance depends on the expert choice of the circle region parameters. In addition, increasing the complexity of the LMIs causes increased number of cases when the LMIs solver does not find a common positive definite matrix $P$ (especially in the case of more complex fuzzy models with a higher number of $r$ rules). Therefore, the procedure where the pole position definition is performed outside the LMI solution is more advantageous one, where the elements of the penalty matrices $Q$ and $R$ of the performance function $J$ of the corresponding LQC (linear quadratic control) problem are optimized separately. One possibility is to find optimal values of penalizing matrices $Q$ and $R$ by a suitable optimization procedure minimizing the trace of matrix $P$. A genetic algorithm can be used, whose chromosome is formed by elements of matrices $Q$ and $R$ with fitness function $F=\operatorname{trace}(P)$. The effectiveness of such solution was proved also for fuzzy

\footnotetext{
1 VSB-Technical University of Ostrava, FEI, Department. of Cybernetics and Biomedical Engineering, Ostrava 70300, Czech Republic, 2 Slovak University of Technology in Bratislava, FEI, Institute of Automotive Mechatronics, Bratislava 81219 , Slovakia, * Corresponding author: danica.rosinova@stuba.sk
} 
LQC of inverted pendulum with elevation [9]. In other case, where a satisfactory result was not achieved, a new shape of the fitness function has been designed that directly includes the response shape parameters. This feature has shown greater effectiveness and is presented and analysed in this paper.

The paper is organized as follows. Section 2 recalls principles of modelling and control of nonlinear systems by Takagi-Sugeno method (Section 2.1). It introduces the T-S fuzzy model as a universal approximator of real continuous functions. Parallel Distributed Controller (PDC) method for synthesis of nonlinear T-S fuzzy controller deriving control rules from corresponding rules of fuzzy T-S model is presented. Section 2.2 deals with the Linear Matrix Inequalities (LMIs) framework which is used to solve optimization problems namely Lyapunov stability problem, PDC design based on stability constraints transformable into LMIs and Linear Quadratic Control (LQR) procedure minimizing the quadratic error and control action energy. Section 3 is devoted to quadratic stability problem and stabilization of T-S systems using Lyapunov method. The conditions of global asymptotic stability proposed in [10] for continuous closed-loop T-S fuzzy systems which satisfy the Lyapunov inequalities are recalled. The essence is in transforming stability conditions into LMIs. Section 4 deals with the properties and behaviour of quadratically stabilizing control designed for T-S systems. Besides the system stability, in practice the controller must also meet other performance requirements. Therefore, an optimal LQR controller is designed to minimize state transient behaviour and control action energy. The paper main contribution is in further improvement of the response, by optimization of $Q$ and $R$ matrices performed using genetic algorithm and in defining a new fitness function to further improve the closed-loop system response according to prescribed requirements. To define the desired dynamics of the response in terms of the mechanics and kinematics of the considered controlled system - quadrotor, a new shape of the fitness function has been designed. The important contribution is in application case study presented in Section 5. An example of stabilization and control of quadrotor is shown via transient responses of its rotation and translation control. Quadrotor case-study explains the principles of optimization of transient responses by searching for optimal values of weighting matrices of LQR optimization procedure. We present concrete simulation results of optimization of the quadrotor rotation and translation system using genetic algorithm by minimizing the trace of the common $\mathrm{P}$ matrix. The properties of a new proposed fitness function are discussed and documented by simulation results. Finally, in Section 6, conclusion and outline of further research are given.

\section{Nonlinear system control problem and preliminaries}

Consider a general nonlinear continuous time dynamical system, described by

$$
\dot{\mathbf{x}}=f(\mathbf{x}, \mathbf{u}),
$$

where $\mathbf{x}$ is $n$-dimensional state vector and $\boldsymbol{u}$ is $m$ dimensional input vector. The control design aim is to find the control law $\mathbf{u}(x)$ so that the corresponding closed-loop system is stable with optimal performance with respect to the defined performance criterion. In this paper, quadratic criterion known from LQ approach is considered as well as additional fitness function to tune the system response.

To cope with system nonlinearity, we use TakagiSugeno fuzzy model which enables to decompose a nonlinear system into a set of linear subsystems. More details on T-S fuzzy modelling and control are recalled in Section 2.1, the efficient computational approach based on LMI formulation is summarized in Section 2.2

\subsection{Fuzzy T-S modelling and control}

This section presents a linguistic description of multivariate space of input variables using antecedents of the set of IF-THEN rules and definition of a linear state model in their consequences. It explains the principle of modelling a nonlinear system by a set of linear submodels. The method of back aggregation of partial linear submodels into the final global nonlinear model is introduced.

Many of complex nonlinear systems can be expressed in some form of mathematical model locally or as an aggregation of a set of linear mathematical models. Takagi and Sugeno have proposed a fuzzy model to describe the complex dynamic model representing a complex singleinput multi- output system, which includes both local analytic linear models and fuzzy membership and fuzzy logic functions [4]. Specifically, the continuous-time T-S fuzzy dynamic model is described by fuzzy IF-THEN rules, which locally represent linearized input-output relations of non-linear systems. Fuzzy subspaces are defined by conjunction of fuzzy-logic statements considering the actual value of input language variables.

As outlined above, T-S model can be used for effective decomposition of $n$-dimensional non-linear system into $r$-linear subsystems. To formalize linguistic values of language variables, fuzzy sets are used and to derive the output language variable value, fuzzy logic laws are applied. Nonlinear system (1) can be effectively modelled by fuzzy "blending of equivalent linear systems in different operating regions using T-S fuzzy model. A T-S fuzzy model is composed of $r$ rules, where the $i$-th rule has the following form

$$
\begin{aligned}
R_{i}: & \operatorname{IF}\left(x_{1} \text { is } M_{i 1}\right) \text { and } \ldots \text { and }\left(x_{n} \text { is } M_{i n}\right) \\
& \text { THEN } \dot{x}=A_{i} x+B_{i} u,
\end{aligned}
$$

where $M_{i j}$ is $j$-th fuzzy term of the $i$-th rule, $i=1,2, \ldots, r, j=1,2, \ldots, n$ and $x=\left[x_{1}, x_{2}, \ldots, x_{n}\right]^{\top}$. 
Let $w_{i}(x)=\prod_{j=1}^{n} \mu_{i j}\left(x_{j}\right)$ where $\mu_{i j}\left(x_{i}\right)$ is a membership function of actual value $x_{j}$ in a fuzzy term $M_{i j}$.

The corresponding fuzzy model around this operating point is constructed as the weighted average of the local models and has the form

$$
\dot{x}=\sum_{i=1}^{r} \bar{w}_{i}(x)\left(A_{i} x+B_{i} u\right),
$$

where

$$
\bar{w}_{i}(x)=\frac{w_{i}(x)}{\sum_{i=1}^{r} w_{i}(x)}, \quad \sum_{i=1}^{r} \bar{w}_{i}(x)=1,
$$

is the normalized value of $w_{i}(x)$ which represents the weighting coefficient of $i$-th local model in (2). Thus, the T-S model representation of the nonlinear system is informative in terms of local dynamics.

Once a nonlinear system is described by a T-S fuzzy model, the following procedure can be applied to design a stable optimal PDC closed-loop control system. The main idea of PDC controller design is to derive control rules from corresponding rules of fuzzy T-S model. A partial linear controller is defined for each rule. The resulting fuzzy controller, which is generally nonlinear, is a fuzzy aggregation of the individual linear controllers. The PDC fuzzy controller for the fuzzy system (1) can be derived as follows $[6]$

$$
\begin{aligned}
R_{i}: & \operatorname{IF}\left(x_{1} \text { is } M_{i 1}\right) \text { and } \ldots \text { and }\left(x_{n} \text { is } M_{i n}\right) \\
& \operatorname{THEN} u(t)=-K_{i} x, i=1, \ldots, r
\end{aligned}
$$

which has a linear state controller in its sequence. The overall PDC nonlinear state controller is then in the form

$$
u=-\sum_{i=1}^{r} \bar{w}_{i}(x) K_{i} x, \quad i=1, \ldots, r .
$$

The PDC scheme stabilizing the T-S fuzzy model is a design framework comprising a control algorithm conforming to a stability condition. The goal is to find appropriate $K_{i}$ gains that ensure stability of the corresponding closed loop system

$$
\dot{x}=\sum_{i=1}^{r} \bar{w}_{i}(x)\left[A_{i}-B_{i} K_{i}\right] x .
$$

The proposed design procedure uses stability conditions based on Lyapunov function, formulated through linear matrix inequalities (LMI).

\subsection{Linear matrix inequalities (LMI) technique}

Linear Matrix Inequalities belong to the semi-definite programming (SDP) that are convex problems. The advantage of SDP is the polynomial time of global minimum computation [2]. Linear matrix inequality is in the form

$$
F(x)=F_{0}+\sum_{i=1}^{m} x_{i} F_{i}>0
$$

where $x(t)=\left[x_{1}(t), \ldots, x_{m}(t)\right]^{\top}$ is the search variable vector and $F_{i}=F_{i}^{\top} \in R^{n \times n}, i=0, \ldots, m$ are known matrices. Inequality means that $F(x)$ must be positive definite, $i e$ all its eigenvalues are positive. The basic LMI solution problem is to find $x^{\text {feas }}$ so that (4) holds or to determine that a solution to LMIs is not feasible. It is a convex feasibility problem that can be efficiently solved by convex optimization algorithms such as interior point methods [7], in polynomial time.

The LMI approach is widely used to solve many convex optimization problems. In control, Lyapunov stability problem ( $\mathrm{P}$ problem) for multimodel, or robust control can be formulated by LMIs and effectively solved by convex optimization techniques. Linear matrix inequalities are also an important tool for fuzzy control of nonlinear systems. Widely used PDC design employs stability conditions transformable into LMIs to obtain the controller gains. Eventually, one of the standard and effective application of LMIs technique is formalization of Linear Quadratic Control (LQR) optimization procedure.

\section{Stability of T-S systems}

Stability of T-S fuzzy model described in Section 2.1 can be analysed using the Lyapunov direct method based on Lyapunov function, which can be interpreted as a measure of system energy. If the total energy of the system decreases continuously with time, then the system tends to steady state and is stable in the Lyapunov sense $[4,5]$. The classical quadratic Lyapunov function can be used to analyse the stability of nonlinear MIMO systems, specifically fuzzy T-S systems.

A system is quadratically stable if there exists a quadratic Lyapunov function $V(x(t))=x^{\top}(t) P x(t)$ such that the following conditions hold on a system solution

$$
\begin{aligned}
& V(x(t))>0, \quad \forall x(t) \neq 0 \Longleftrightarrow P>0, \\
& \dot{V}(x(t))<0, \quad \forall x(t) \neq 0
\end{aligned}
$$

where $x=\left(x_{1}, \ldots, x_{n}\right)^{\top}$ is a vector of state variables and $P$ is a symmetric square matrix of order $n$.

Sufficient condition of global asymptotic stability of open-loop T-S fuzzy system

$$
\dot{x}(t)=\sum_{i=1}^{r} \bar{w}_{i}(x) A_{i} x(t),
$$

according to Lyapunov stability condition, is the existence of a positive definite matrix $P$ which satisfies inequality

$$
A_{i}^{\top} P+P A_{i}<0, i=1, \ldots, r
$$


where the matrix $P$ is common for all submodels of the T-S model $[2,4,5]$.

The closed-loop T-S fuzzy system (3) is globally asymptotically stable if there exists a common positive definite matrix $\mathrm{P}$ which satisfies the following Lyapunov inequalities

$$
\begin{gathered}
\left(A_{i}-B_{i} K_{i}\right)^{\top} P+P\left(A_{i}-B_{i} K_{i}\right)<0, \\
G_{i j}^{\top} P+P G_{i j}<0, \quad i<j \leq r,
\end{gathered}
$$

where $G_{i j}$ is defined as $G_{i j}=A_{i}-B_{i} K_{j}+A_{j}-B_{j} K_{i}$. Using the change of variables $Y=P^{-1}, X_{i}=K_{i} Y$ inequalities (6) can be rewritten as LMIs

$$
\begin{gathered}
Y A_{i}^{\top}+A_{i} Y-B_{i} X_{i}-X_{i}^{\top} B_{i}^{\top}<0, i=1, \ldots, r \\
Y\left(A_{i}+A_{j}\right)^{\top}+\left(A_{i}+A_{j}\right) Y-\left(B_{i} X_{j}-B_{j} X_{i}\right)- \\
\left(B_{i} X_{j}-B_{j} X_{i}\right)^{\top}<0, i<j \leq r .
\end{gathered}
$$

If the inequalities (7) are feasible with a positive definite solution, the stability of the closed loop system (3) is guaranteed. However, in practice the controller must also meet other performance requirements.

\section{Optimal performance of T-S system}

In this section, the LQR optimal control design for T-S system (5) is briefly recalled and further improved by: i) optimization of cost function parameters (matrices $Q$ and $R$ ); ii) introducing a new fitness function to shape the closed-loop system response according to the required maximal overshoot and rise time.

\section{$L Q R$ optimal control}

Below, the problem of designing a stable closed-loop system containing a state feedback fuzzy controller is studied. Firstly, the optimal LQR controller is designed to minimize quadratic error (nonzero state variables) and control action energy. Recall that in this case, for system (5) we minimize the following quadratic cost function, $[9,10]$

$$
J=\frac{1}{2} \int_{0}^{\infty}\left(x^{\top}(t) Q x(t)+u^{\top} R u(t)\right) \mathrm{d} t,
$$

where symmetric matrices $Q=Q^{\top}>0, R=R^{\top}>0$ are the penalty matrices defining the weights of the quadratic error and the control action respectively. Next, these matrices represent the design parameters to optimize the state feedback controller.

The control design aim is to find the control law $u=f(x)$, which ensures the minimization of the function $J \rightarrow$ min (ie placing the closed-loop poles in the optimal position as a result of minimizing $J$ ). The control law can be written as

$$
u(t)=-R^{-1} B^{\top} P x(t)=-K x(t),
$$

thus, the control gain matrix is given as

$$
K=R^{-1} B^{\top} P
$$

Control law (9) and (10), guarantees to keep the output as close as possible to the desired value with minimum control energy simultaneously.

Matrix $P$ can be calculated off-line. The symmetric matrix $P$ is the unique solution of the algebraic Riccati equation [11]

$$
P A+A^{\top} P+Q-P B R^{-1} B^{\top} P=0 .
$$

Regarding $u(t)$ from (9), we have a closed-loop system state transition equation

$$
\dot{x}(t)=A x(t)+B u(t)=(A-B K) x(t),
$$

with closed-loop system transient matrix

$$
\bar{A}=A B K,
$$

in which the eigenvalues of $\bar{A}$ are the closed-loop poles. Regarding the closed-loop system we can write the cost function $J(8)$ as

$$
J=\frac{1}{2} \int_{0}^{\infty} x^{\top}(t) Q x(t)+x^{\top} K^{\top} R K x(t) \mathrm{d} t .
$$

Considering

$$
\bar{Q}=Q+K^{\top} R K
$$

we obtain the well-known Lyapunov equation

$$
\overline{A^{\top}} P+P \bar{A}=-\bar{Q} .
$$

The existence of a positive definite solution $P$ to (11) ensures that the closed system is asymptotically stable and quadratically optimal at the same time.

The optimality condition is

$$
(A-B K)^{\top} P+P(A-B K)+Q+K^{\top} R K=0,
$$

where $Q \geq 0, R>0$.

Analogically to stability conditions (6), we now can rewrite (12) for T-S closed loop system as a set of LMIs (13). According to LQ theory, the solution of these inequalities will be an upper bound on the performance measure $J$ and we can reach $\min \{J\}$ by minimizing that upper bound [12]

$$
\begin{gathered}
\left(A_{i}-B_{i} K_{i}\right)^{\top} P+P\left(A_{i}-B_{i} K_{i}\right)+Q+ \\
\sum_{i=1}^{r} K_{i}^{\top} R K_{i}<0, \\
G_{i j}^{\top} P+P G_{i j}+Q+\sum_{i=1}^{r} K_{i}^{\top} R K_{i}<0,
\end{gathered}
$$


where $j=1, \ldots, r, i<j<r$. Substituting $Y=P^{-1}$ and using denotation

$$
\begin{aligned}
N_{i}= & \left(Y A_{i}^{\top}+A_{i} Y\right)-B_{i} X_{i}-X_{i}^{\top} B_{i}^{\top} \\
T_{i j}= & Y\left(A_{i}+A_{j}\right)^{\top}+\left(A_{i}+A_{j}\right) Y \\
& -\left(B_{i} X_{j}+B_{j} X_{i}\right)-\left(B_{i} X_{j}+B_{j} X\right)^{\top},
\end{aligned}
$$

we can write

$$
\begin{aligned}
& N_{i}+Y Q Y+\sum_{i=1}^{r} X_{i}^{\top} R X_{i}<0, \\
& T_{i j}+Y Q Y+\sum_{i=1}^{r} X_{i}^{\top} R X_{i}<0
\end{aligned}
$$

where $i=1, \ldots, r, i<j \leq r$.

Denoting $S_{i j}=\left(B_{i} X_{j}+B_{j} X_{i}\right)$, we obtain

$$
T_{i j}=Y\left(A_{i}+A_{j}\right)^{\top}+\left(A_{i}+A_{j}\right) Y-S_{i j}-S_{j}^{\top} .
$$

All corresponding LMIs can be summarized as [7]

$$
\begin{aligned}
& {\left[\begin{array}{ccccc}
N_{i} & Q^{1 / 2} Y & X_{1}^{T} R^{1 / 2} & \ldots & X_{r}^{T} R^{1 / 2} \\
Q^{1 / 2} Y & -I_{m} & 0 & \ldots & 0 \\
R^{1 / 2} X_{1} & 0 & -I_{m} & \ldots & 0 \\
\cdot & \cdot & \cdot & \ldots & \cdot \\
. & \cdot & . & \ldots & \cdot \\
R^{1 / 2} X_{r} & 0 & 0 & \ldots & -I_{m}
\end{array}\right]<0,} \\
& {\left[\begin{array}{ccccc}
T_{i j} & Q^{1 / 2} Y & X_{1}^{T} R^{1 / 2} & \ldots & X_{r}^{T} R^{1 / 2} \\
Q^{1 / 2} Y & -I_{m} & 0 & \ldots & 0 \\
R^{1 / 2} X_{1} & 0 & -I_{m} & \ldots & 0 \\
\cdot & . & \cdot & \ldots & . \\
R^{1 / 2} X_{r} & 0 & 0 & \ldots & -I_{m}
\end{array}\right]<0}
\end{aligned}
$$

where $i=1, \ldots, r i<j \leq r$. If LMIs (15) are feasible, we can calculate the controller gains as

$$
K_{i}=X_{i} Y^{-1}
$$

and the corresponding control law is

$$
u=\sum_{i=1}^{r} \bar{w}_{i}(x) K_{i} x, \quad i=1, \ldots, r .
$$

As mentioned above, the LQR closed-loop system is asymptotically stable in the Lyapunov sense.

\section{Transient performance modification}

In many practical cases, it is necessary to determine the transient response of a control system by rise time and appropriate settling time. For example, such problems may arise in solving the cooperation of movement of individual agents in the collaborative systems.

The LQR optimization method aims at minimizing the quadratic performance index for given values of $Q$ and
$R$. Obviously, the parameters of transient responses can be influenced by the values of the weighting matrices $Q$ and $R$ and corresponding eigenvalues of the matrix $P$. Therefore, matrices $Q$ and $R$ are often tuned manually: expertly by trial-and-error method. However, the optimal values of the weighting matrices can be systematically found by a suitable optimization procedure, as genetic algorithm (GA) [13]. Then, the chromosome is defined as

$$
\left[q_{11}, q_{22}, \ldots, q_{n n}, r_{11}, r_{22}, \ldots, r_{m m}\right]
$$

where

$$
\begin{aligned}
Q & =\operatorname{diag}\left[q_{11}, q_{22}, \ldots, q_{n n}\right] \\
R & =\operatorname{diag}\left[r_{11}, r_{22}, \ldots, r_{m m}\right],
\end{aligned}
$$

and the fitness function has the shape [13]

$$
F^{\text {orig }}=\operatorname{trace}(P) \rightarrow \min .
$$

The results presented in Section 5 suggest effectiveness of an additional optimization of the weighting matrices $Q$ and $R$.

\section{Fitness function modification}

The fitness function (16) is only suitable to ensure, for example, an aperiodic response. Therefore, a new shape of the fitness function is designed here, which directly reflects the response parameters. The relevant values are the maximum overshoot and the rise time which is determined as the time between 10 and $90 \%$ of the desired output value. The fitness function is modified to consider both maximum overshoot and the rise time and/or settling time eventually

$$
F^{\text {modif }}=w_{t}\left(t_{r}-t_{r}^{\text {des }}\right)^{2}+w_{z} z_{\text {over }}^{2} \rightarrow \min ,
$$

where $t_{r}(\mathrm{~s})$ is a rise time, $t_{r}^{\text {des }}(\mathrm{s})$ is the required rise time, $z_{\text {over }}(\mathrm{m})$ is overshoot, $w_{t}$ is rise time weighting coefficient and $w_{z}$ is overshoot weighting coefficient. The square of difference between desired and actual rise time ensures fitness function differentiability. To ensure consistent influence of the monitored parameters, we also consider squared overshoot. In each iteration step of the genetic algorithm procedure the values of matrices $Q$ and $R$ are entered, the value of appropriate rise time and overshoot is obtained by simulation and the fitness function convergence to zero is required.

The optimization of the $Q$ and $R$ matrix elements takes place in off-line mode. Therefore, the time-consuming genetic algorithm procedure does not limit the controller implementation.

The effectiveness of the proposed fitness function is shown in the next section via optimization of quadrotor rotation and translation system responses. 


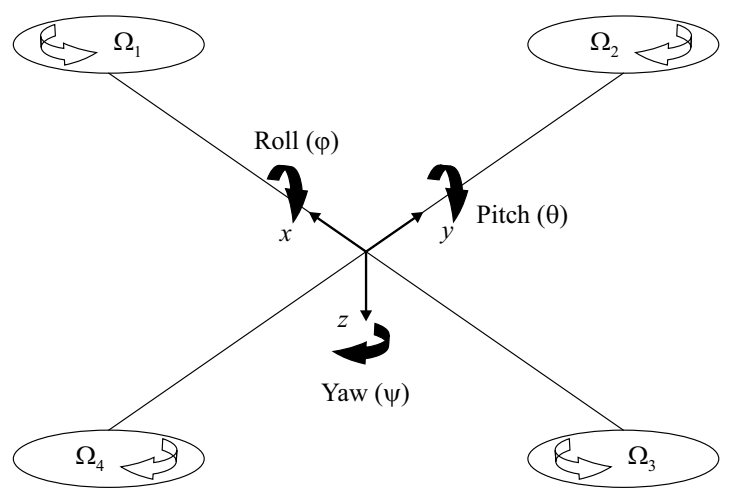

Fig. 1. Quadrotor concept (source: $[14]$ )

\section{Quadrotor control design: simulation results and discussion}

This section presents a case study: a quadrotor nonlinear system control, stabilization and optimization to illustrate the results of the proposed fuzzy controller design method. A quadrotor consists of four motors and four propellers, each located in position as shown in Fig. 1. Propellers 1 and 3 rotate in the same direction while propellers 2 and 4 rotate in an opposite direction [14].

The quadrotor is a 6 DOF device, thus 6 variables are used to express its position in space $(x, y, z, \varphi, \theta$ and $\psi)$. Variables $x, y$ and $z$ represent the distances of the quadrotor center of gravity along the $X, Y$ and $Z$ axis respectively from an Earth fixed inertial frame. The roll angle is represented by $\varphi$ which is the angle about the $X$-axis, $\theta$ is the pitch angle about the $Y$-axis, while $\psi$ is the yaw angle about the $Z$-axis (Fig. 1 ). Rotor speeds $\Omega_{i}$ of each motor are indicated as well.

To handle the quadrotor, the rotation and translation control systems are used as in [14]. The rotational system provides the control of the angles $\varphi, \theta$ and $\psi$; to control the $Z$ position of the quadrotor (height), a translation system is designed. Thus, fuzzy controller proposed in previous sections is designed to control rotation as well as translation along $\mathrm{z}$ (height) movements. On the other hand, to control the position in $X$ and $Y$ axes, a manually operated joystick is used in our example to fully determine the position of the quadrotor. System states $\Phi, \dot{\Phi}, \Theta, \dot{\Theta}, \Psi, \dot{\Psi}, Z, \dot{Z}$ are represented by symbols $x_{1}, x_{2}, x_{3}, x_{4}, x_{5}, x_{6}, x_{7}, x_{8}$. Control inputs correspond to four motor outputs (torques).

We propose the overall control system consisting of two modules: rotational control subsystem and translational control subsystem (to control the height).

\section{Rotational subsystem}

Defining the six rotational states $x_{a}=\left[x_{1}, x_{2}, x_{3}, x_{4}\right.$, $\left.x_{5}, x_{6}\right]^{\top}$ and three control inputs $U_{a}=\left[U_{2}, U_{3}, U_{4}\right]^{\top}$, the rotational subsystem is expressed by the following matrix state equation corresponding to a linearized quadrotor model

$$
\begin{array}{r}
\dot{x}_{a}=\left[\begin{array}{cccccc}
0 & 1 & 0 & 0 & 0 & 0 \\
0 & -e_{1} & 0 & a_{1} z_{2}-a_{2} z_{3} & 0 & 0 \\
0 & 0 & 0 & 1 & 0 & 0 \\
0 & a_{3} z_{2}-a_{4} z_{3} & 0 & -e_{2} & 0 & 0 \\
0 & 0 & 0 & 0 & 0 & 1 \\
0 & a_{5} z_{1} & 0 & 0 & 0 & -e_{3}
\end{array}\right] x_{a} \\
+ \\
+\left[\begin{array}{ccc}
0 & 0 & 0 \\
b_{1} & 0 & 0 \\
0 & 0 & 0 \\
0 & b_{2} & 0 \\
0 & 0 & 0 \\
0 & 0 & b_{3}
\end{array}\right] U_{a},
\end{array}
$$

where $a_{1}=I_{y y}-I_{z z} / I_{x x}, a_{2}=J_{r} / I_{x x}, a_{3}=I_{z z}-$ $I_{x x} / I_{y y}, a_{4}=J_{r} / I_{y y}, a_{5}=I_{x x}-I_{y y} / I_{z z}, b_{1}=L / I_{x x}$, $b_{2}=L / I_{y y}, b_{3}=L / I_{z z}, e_{1}=K_{a x} / I_{x x}, e_{2}=K_{a y} / I_{y y}$, $e_{3}=K_{a z} / I_{z z}$.

The values of quadrotor parameters are the following: quadrotor mass $m_{q}=0.486 \mathrm{~kg}, L=0.255 \mathrm{~m}$, gravitational acceleration $g_{r}=9.81 \mathrm{~m} / \mathrm{s}^{2}$, inertia $J_{r}=3.35 \times$ $10^{-5} \mathrm{~kg} \mathrm{~m}^{2}$, moments of inertia $I_{x x}=3.82 \times 10^{-5} \mathrm{~kg} \mathrm{~m}^{2}$, $I_{y y}=3.82 \times 10^{-5} \mathrm{~kg} \mathrm{~m}^{2}, I_{z z}=7.65 \times 10^{-5} \mathrm{~kg} \mathrm{~m}^{2}, K_{a x}=$ $5.567 \times 10^{-4}, K_{a y}=5.567 \times 10^{-4}, K_{a z}=6.354 \times 10^{-4}$, $K_{r z}=0.048$.

The variables in (18) are defined as $z_{1}=x_{4}, z_{2}=x_{6}$ and $z_{3}=\Omega_{r}$. Here, $\Omega_{r}=-\Omega_{1}+\Omega_{2}-\Omega_{3}+\Omega_{4}$ due to a torque compensation. These variables are used as inputs in the fuzzy T-S model (19) assuming the ranges $x_{4} \in[-0.5,0.5] \mathrm{rad} / \mathrm{s}, x_{6} \in[-0.5,0.5] \mathrm{rad} / \mathrm{s}$ and $\Omega_{r} \in$ $[-4,4] \mathrm{rad} / \mathrm{s}$.

To design a T-S fuzzy model, we define two language values represented by membership functions named "Positive", "Negative" for both input variables $z_{1}, z_{2}$ and "Above" and "Below" for variable $z_{3}$. The appropriate membership functions are of triangular shape with braking points defined as follows: $\mathrm{NE}\left[\begin{array}{lll}-0.5 & -0.5 & 0.5\end{array}\right]$, $\mathrm{PO}\left[\begin{array}{lll}-0.5 & 0.5 & 0.5\end{array}\right], \mathrm{BE}\left[\begin{array}{lll}-4.0 & -4.0 & 4.0\end{array}\right]$ and $\mathrm{AB}\left[\begin{array}{lll}-4.0 & 4.0 & 4.0\end{array}\right]$ respectively.

To divide the space of the input variables, defining the linearized model, into eight appropriated subspaces we propose rotational T-S fuzzy model consisting of eight IF-THEN rules

$$
\text { IF Then }
$$

$R 1$ : ( $z_{1}$ is $\left.P O\right)$ and $\left(z_{2}\right.$ is $\left.N E\right)$ and $\left(z_{3}\right.$ is $\left.A B\right) \quad \dot{x}_{a}=A_{a 1} x+B_{a} U_{a}$ $R 2$ : ( $z_{1}$ is $\left.P O\right)$ and $\left(z_{2}\right.$ is $\left.P O\right)$ and $\left(z_{3}\right.$ is $\left.A B\right) \quad \dot{x}_{a}=A_{a 2} x+B_{a} U_{a}$ $R 3$ : ( $z_{1}$ is $\left.N E\right)$ and $\left(z_{2}\right.$ is $\left.P O\right)$ and ( $v_{3}$ is $\left.A B\right) \quad \dot{x}_{a}=A_{a 3} x+B_{a} U_{a}$ $R 4$ : ( $z_{1}$ is $\left.N E\right)$ and $\left(z_{2}\right.$ is $\left.N E\right)$ and $\left(z_{3}\right.$ is $\left.A B\right) \dot{x}_{a}=A_{a 4} x+B_{a} U_{a}$ R5 : ( $z_{1}$ is PO) and ( $z_{2}$ is $\left.P O\right)$ and $\left(z_{3}\right.$ is $\left.B E\right) \quad \dot{x}_{a}=A_{a 5} x+B_{a} U_{a}$ $R 6$ : ( $z_{1}$ is PO) and $\left(z_{2}\right.$ is $\left.N E\right)$ and $\left(z_{3}\right.$ is $\left.B E\right) \quad \dot{x}_{a}=A_{a 6} x+B_{a} U_{a}$ $R 7$ : ( $z_{1}$ is $\left.N E\right)$ and $\left(z_{2}\right.$ is $\left.P O\right)$ and ( $z_{3}$ is $\left.B E\right) \quad \dot{x}_{a}=A_{a 7} x+B_{a} U_{a}$ $R 8$ : $\left(z_{1}\right.$ is $\left.N E\right)$ and $\left(z_{2}\right.$ is $\left.N E\right)$ and $\left(z_{3}\right.$ is $\left.B E\right) \dot{x}_{a}=A_{a 8} x+B_{a} U_{a}$

State-space matrices of rotational fuzzy model (19) are in the form 


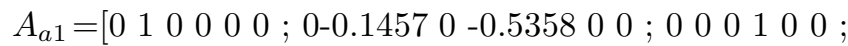
$00.46620-0.145700 ; 000001 ; 00000-0.0830]$

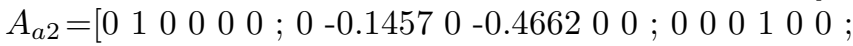
$00.53580-0.145700 ; 000001 ; 00000-0.0830]$

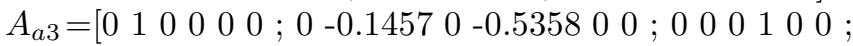
$00.46620-0.145700 ; 000001 ; 00000-0.0830]$

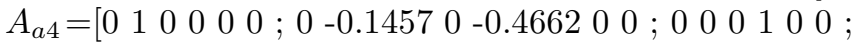
$00.53580-0.145700 ; 000001 ; 00000-0.0830]$

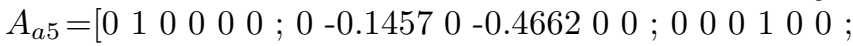
$00.53580-0.145700 ; 000001 ; 00000-0.0830]$

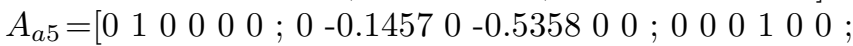
$00.46620-0.145700 ; 000001 ; 00000-0.0830]$

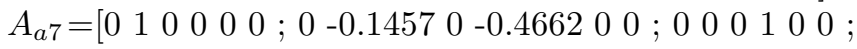
$00.53580-0.145700 ; 000001 ; 00000-0.0830]$

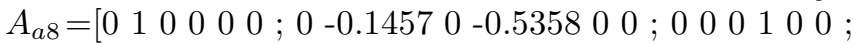
$00.46620-0.145700 ; 000001 ; 00000-0.0830]$

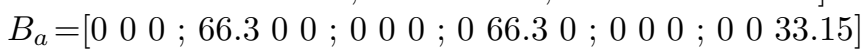

\section{Translational subsystem}

Defining variables $x_{b}=\left[x_{7}, x_{8}\right]^{\top}$ and $e_{6}=K_{r z} / m_{q}$ we have translational subsystem model in the form

$$
\dot{x}_{b}=\left[\begin{array}{cc}
0 & 1 \\
0 & e_{6}
\end{array}\right] x_{b}+\left[\begin{array}{c}
0 \\
-\frac{z_{4}}{m_{q}}
\end{array}\right] U_{1}+\left[\begin{array}{c}
0 \\
g_{r}
\end{array}\right] .
$$

The variable $z_{4}$ is a non-linear term defined by $z_{4}=$ $\cos x_{1} \cos x_{3}$. The ranges are assumed $x_{1} \in[-\pi / 3, \pi / 3]$, $x_{3} \in[-\pi / 3, \pi / 3] \mathrm{rad}$. This variable is used as input in the fuzzy T-S model (21).

Now, we define two language values for both input variables represented by membership functions named "Maximum", "Minimum". The appropriate membership functions are of triangular shape with breaking points MN [- 0.5 - 0.50 .5$]$ and MX [- 0.50 .50 .5$]$ respectively.

To divide the space of the input variables into two appropriated subspaces we propose translational T-S fuzzy model (21) consisting of two IF-THEN rules

$$
\begin{aligned}
& R 1: \operatorname{IF}\left(z_{4} i s M X\right) \text { THEN } \dot{x}_{b}=A_{b} x_{b}+B_{b 1}+G \\
& R 2: \operatorname{IF}\left(z_{4} i s M N\right) \text { THEN } \dot{x}_{b}=A_{b} x_{b}+B_{b 2}+G .
\end{aligned}
$$

State-space matrices of translational fuzzy model (21) are in the form

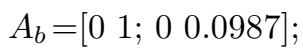

$B_{b 1}=[0 ; 1]$

$B_{b 2}=[0 ; 0.25]$

\section{LMIs feasibility and gain matrix calculation}

Quadrotor rotational and translational subsystem fuzzy controllers were designed by solving LMIs (15) introduced in Section 4. LMI solution was performed by LMI toolbox in Matlab and, alternatively, using SeDuMi solver via YALMIP toolbox, [16,17]. Result of LMI Toolbox [15] - calculation of LMIs feasibility for the rotational system and $Q=\operatorname{diag}(5)$ and $R=\operatorname{diag}(2)$ was: "Could not establish feasibility nor infeasibility. Marginal infeasibility: these LMI constraints may be feasible but are not strictly feasible". In fact, the matrix $P$ was found only as semi-definite and some gain values of $K_{\mathrm{i}}$ were zero. In YALMIP Toolbox and SeDuMi, a feasible solution was found: positive definite matrix $P$ and gain matrices $K_{\mathrm{i}}$

$$
\begin{aligned}
& P=2.48898 .0092-0,3149-0.9332-0,3906-0,8497 \\
& \begin{array}{lllll}
8.0092 & 2.7356 & 0.1013 & 0.2523 & -0,1189-0,2715
\end{array}
\end{aligned}
$$

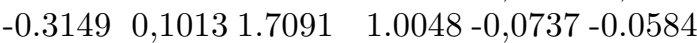

$$
\begin{aligned}
& \begin{array}{lllll}
-0.9332 & 0.2523 & 1.0048 & 3.4230 & -0,1139-0,1837
\end{array} \\
& \begin{array}{lllll}
-0.3906 & -0,1189-0,0737-0,1139 & 1,5992 & 0,7964
\end{array} \\
& \begin{array}{llll}
-0.8497-0.2715 & -0.0584-0,1837 & 0.7964 & 2,3072
\end{array} \\
& K_{1}=0.18270 .1315-0.0527-0.0442-0.0764-0.0509 \\
& \begin{array}{lllll}
-0.1923-0.1355 & 0.3293 & 0.2434 & -0.0830 & -0.0563
\end{array}
\end{aligned}
$$

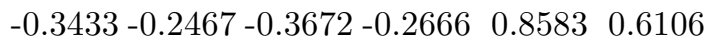

$$
\begin{aligned}
& K_{1}=0.2951 \quad 0.2179-0.1053 \quad 0.0808-0.1249-0.0839
\end{aligned}
$$

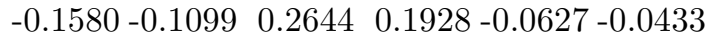

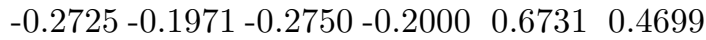

$$
\begin{aligned}
& K_{3}=0.19780 .1430-0.0525-0.0433-0.0887-0.0594 \\
& \begin{array}{llllll}
-0.1981 & -0.1402 & 0.3275 & 0.2421 & -0.0766 & -0.0519
\end{array}
\end{aligned}
$$

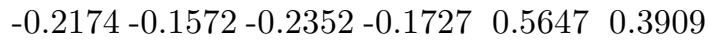

$$
\begin{aligned}
& K_{4}=0.22320 .1621-0.0633-0.0505-0.1031-0.0697
\end{aligned}
$$

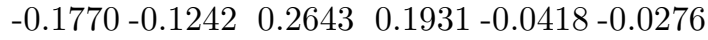

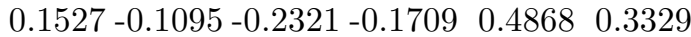

$$
\begin{aligned}
& K_{5}=0.21120 .1532-0.0600-0.0477-0.0941-0.0631
\end{aligned}
$$

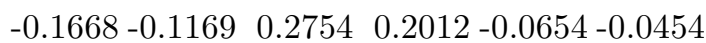

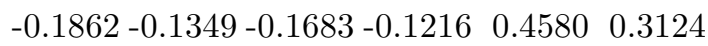

$$
\begin{aligned}
& K_{6}=0.2480 \quad 0.1810-0.0983-0.0780-0.0904-0.0597
\end{aligned}
$$

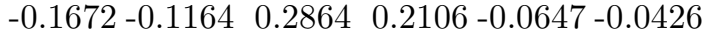

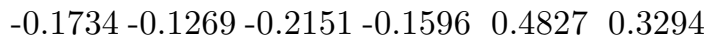

$$
\begin{aligned}
& K_{7}=0.22640 .1640-0.0920-0.0733-0.0826-0.0556
\end{aligned}
$$

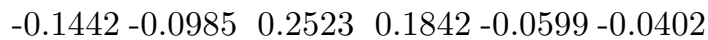

$$
\begin{aligned}
& 0.1374-0.0986-0.1953-0.1435 \quad 0.4301 \quad 0.2924 \\
& K_{8}=0.25520 .1875-0.0984-0.0774-0.0928-0.0603
\end{aligned}
$$

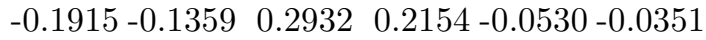

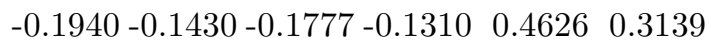

The results of YALMIP Toolbox calculation of LMIs for the translational system and $Q=\operatorname{diag}(5) ; R=\operatorname{diag}(2)-$ a feasible solution was found, matrix $P$ and gain matrices are:

$$
\begin{aligned}
& P=\left[\begin{array}{llll}
9.8395 & 11.5129 ; 11.5129 & 35.5523
\end{array}\right] \\
& K_{1}=0.8020 \quad 2.2308 \\
& K_{2}=1.8472 \quad 4.9833
\end{aligned}
$$

Elements of cost function matrices $Q$ and $R$ were further optimized using genetic algorithm and the fitness function (16) and finally, the novel proposed fitness function (17). The GA parameters were as follows.

GA optimization - chromosome for rotational subsystem: $n=6, m=3$

$$
\left[q_{11}, q_{22}, q_{33}, q_{44}, q_{55}, q_{66}, r_{11}, r_{22}, r_{33}\right]
$$

GA optimization - chromosome for translational subsystem: $n=2, m=1$

$$
\left[q_{11}, q_{22}, r_{11}\right]
$$

The above calculated gain values and $Q$ and $R$ elements values were used to verify the performance of the 


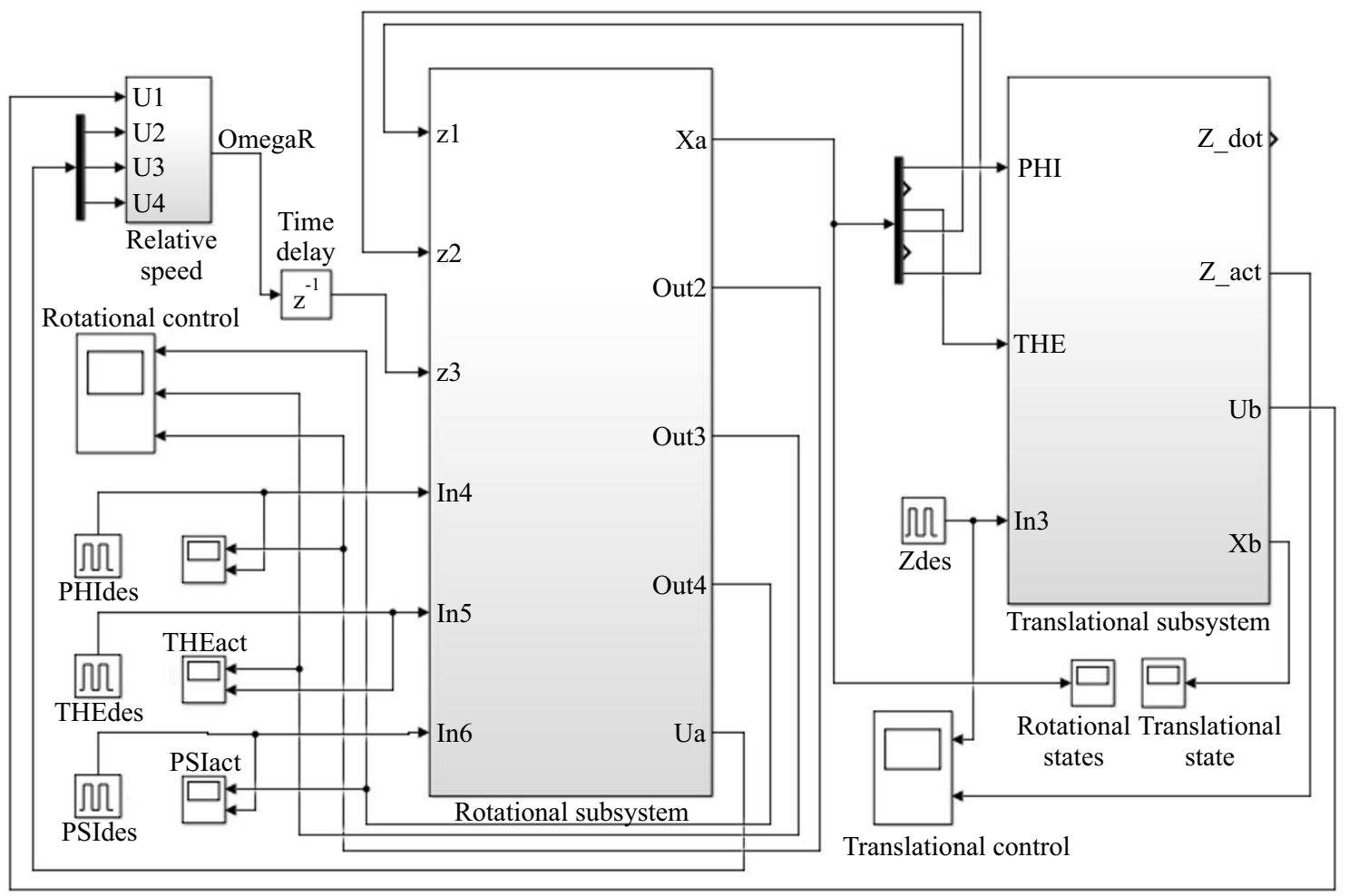

Fig. 2. Quadrotor control scheme in simulink: rotational and translational subsystems

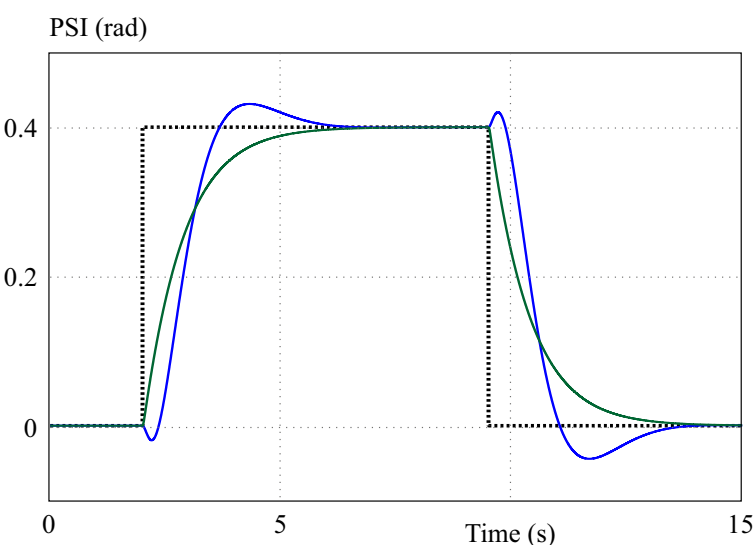

Fig. 3. Step responses for PSI angle control: comparison of LQ control (blue line) and LQ with optimized matrices $Q$ and $R$ (green line)

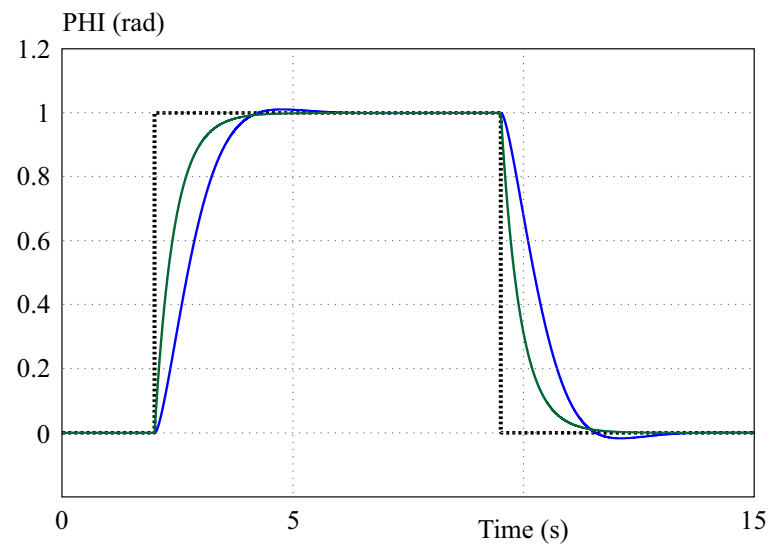

Fig. 5. Step responses for PHI angle control: comparison of LQ control (blue line) and LQ with optimized matrices $Q$ and $R$ (green line)

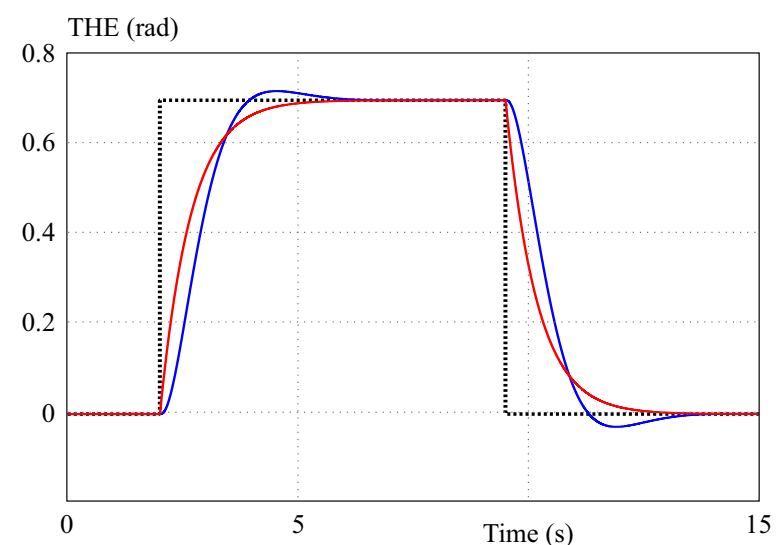

Fig. 4. Step response for the height (translational) control: LQ with optimized matrices $Q$ and $R$ (red line)

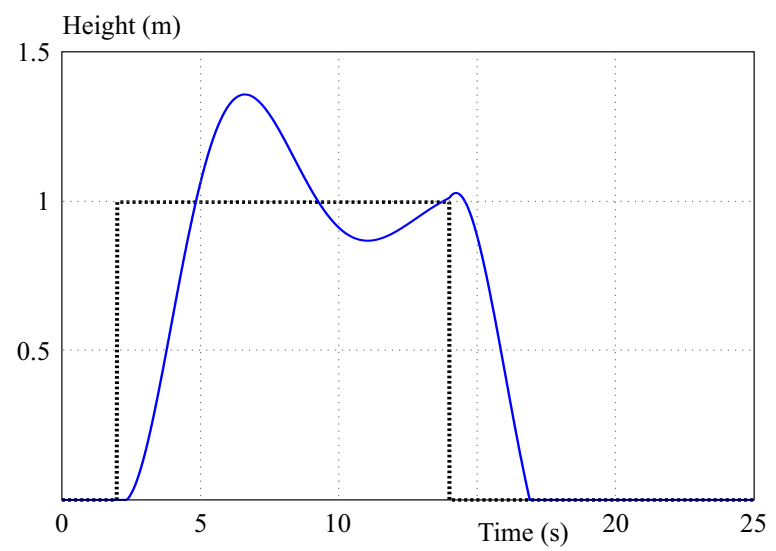

Fig. 6. Step response for the height (translational) control: LQ with optimized matrices $Q$ and $R$ using fitness function (16) 
proposed method of T-S modelling, control and optimization in Matlab and Matlab-Simulink. Figure 2 shows a quadrotor control scheme implementation in Simulink.

Simulation results for quadrotor rotational and translational control received applying only LQR optimization procedure are given in the next figures. The blue lines correspond to the system responses obtained from simulation without additional GA optimization and the red lines show the results obtained with GA optimization using fitness function $F^{\text {orig }}(16)$.

Simulation results corresponding to the PSI angle control are shown in Fig. 3. The control objective is to keep the angle 0.4 radians. Figure 3 shows that the model reaches the desired angle in 7 seconds approximately, with overshoots, on the other hand, the GA optimization eliminates overshoots.

Simulation results corresponding to the angle control are shown in Fig. 4. The control objective is to keep the angle 0.7 radian. Figure 4 shows that the model reaches the desired angle in 7 seconds approximately with an overshoot, the GA optimization provides the overshoot elimination again.

Simulation results corresponding to the PHI angle control are Shown in Fig. 5. The control objective is to keep the angle 1 radian. Figure 5 shows that the model reaches the desired angle in 7 seconds approximately with a small overshoot, however, the GA optimization again eliminates the overshoot.

In conclusion, GA optimization of the rotational motion control with application of fitness function shows fully satisfactory results. On the contrary, if the optimization of the vertical (translational) motion control of the quadrotor is performed in the same manner, the result is fully insufficient - response is oscillating with large overshoots (see Fig. 6).

However, very good result is obtained for the behaviour of translational system which the GA procedure applying the new fitness function $F^{\text {modif }}(17)$. Now, the required height is met without overshoots and a desired rise time tuning is available in addition. The relevant results of translation response parameters tuning are presented in Tab. 1.

An example of the genetic algorithm procedure calculation is given in Fig. 7 .

The corresponding influence of the parameter tuning on the response performance is shown in Fig. 8. Here, the green line corresponds to the desired rise time $0.5 \mathrm{~s}$, the blue line corresponds to desired value $4 \mathrm{~s}$. The simulations are performed for weighting coefficients $w_{t}$ and $w_{z}$ set to 1 .

With our proposed fitness function (17), it is now possible to prescribe the dynamics of the aperiodic response in terms of the mechanics and kinematics of a quadrotor by setting a desired rise time or taking into account the settling time eventually.

\section{Conclusion and future work}

The purpose of this paper is to present an advanced design of Takagi-Sugeno fuzzy controllers for nonlinear systems. We presented a fuzzy PDC controller stable in Lyapunov sense and minimizing the upper bound of a linear quadratic performance measure LQR using the guaranteed cost approach simultaneously. Stability conditions and quadratic performance are guaranteed by solving a corresponding system of linear matrix inequalities LMIs. An additional GA procedure is used to optimize the penalty matrices of LQR scheme. To achieve a relevant performance of transient response, a new form of GA fitness function taking into account the mechanics and kinematics of the real controlled system is proposed. The effectiveness of presented methods is illustrated on quadrotor control system design and investigation. Future research regarding the problems in the consensus control of multiple quadrotor performing the transportation by horizontal displacement is planned. Quadrotor controllers must be designed in order to assure the system stability and quick convergence for the robust achievement to the current task. The off-line and on-line control strategies will be proposed and investigated to ensure their ability to cope with variable dynamic conditions of the system including external disturbances.

\section{Acknowledgments}

Research is supported by the European Regional Development Fund in the Research Centre of Advanced Mechatronic Systems project CZ.02.1.01/0.,0/0.0/ 16_019/0000867 within the Operational Programme Research, Development and Education, and by Slovak Scientific Grant Agency, grant No 1/0745/19.

Table 1. Translation response adjusting

\begin{tabular}{lccccc}
\hline Rise time & Rise time & Overshoot & Penalty matrix & Translational gains & Translational gains \\
desired $(\mathrm{s})$ & $(\mathrm{s})$ & $(\%)$ & $q_{11}, q_{22}, r_{11}$ & $K t 1$ & $K t 2$ \\
0.5 & $0.51(0.12 ; 0.63)$ & 3 & $1.18 \times 10^{2} 13.247331 .32 \times 10^{-4}$ & 25.776379 .85818 & 75.8915125 .88134 \\
1 & $1(0.21 ; 1.21)$ & 1 & 35.227138 .454150 .03638 & 10.349926 .94417 & 27.0372617 .75195 \\
2 & $2(0.34 ; 2.34)$ & 0 & 20.1545414 .832570 .16626 & 4.504955 .46728 & 12.1292414 .67895 \\
3 & $3(0.59 ; 3.59)$ & 0 & 11.1761715 .476210 .69519 & 1.509592 .88451 & 4.455908 .51796 \\
4 & $4(0.82 ; 4.82)$ & 0 & 9.1766223 .588541 .47512 & 0.791432 .02832 & 2.598286 .68103 \\
\hline
\end{tabular}



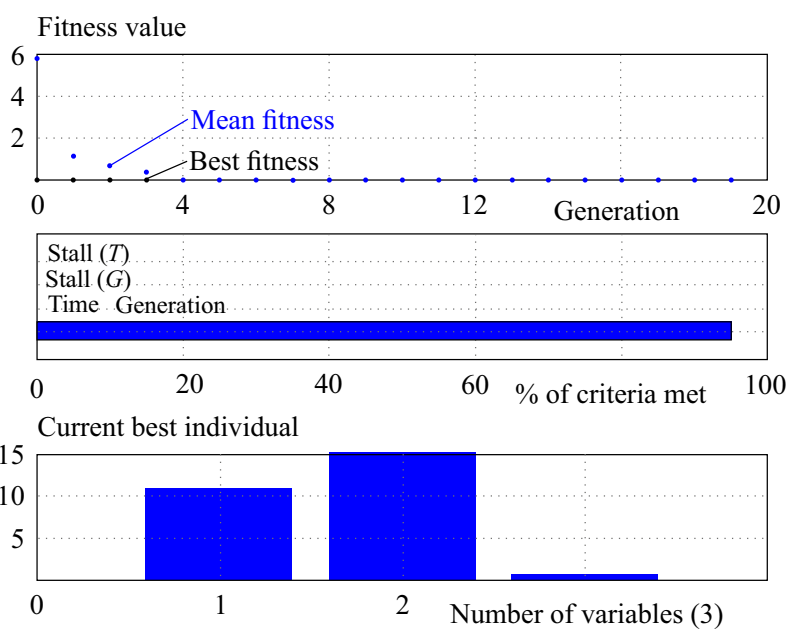

Fig. 7. genetic algorithm calculation

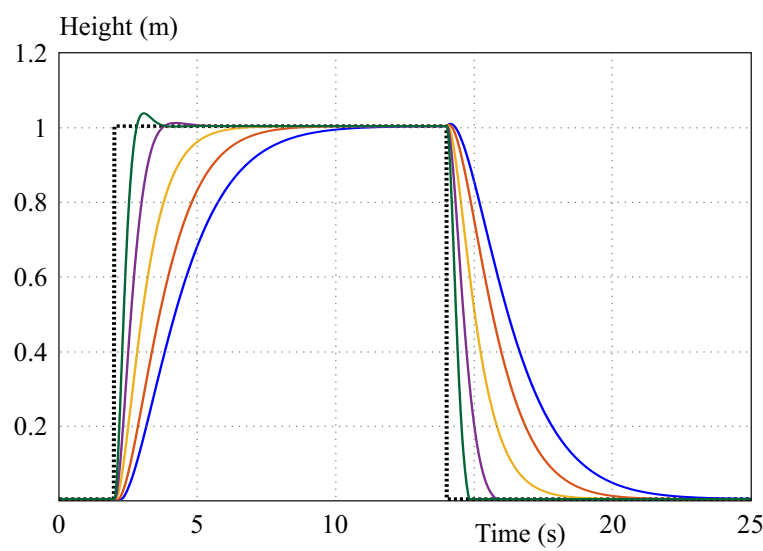

Fig. 8. Step response for the height (translational) control: LQ control with optimized matrices $Q$ and $R$ using the new proposed fitness function (17) for various choices of rise time

\section{REFERENCES}

[1] L. A. Zadeh, "Fuzzy Sets Information and control vol. 8, 1965.

[2] I. Abdelmalek, "Non Linear Systems Control: LMI Fuzzy Approach", PHD thesis, University of Hadj Lakhdar de Batna, Algerie, 2009.

[3] L. X. Wang, and J. M. Mendel, "Fuzzy basis functions, universal approximators and orthogonal least-squares learning", IEEE Transactions on Neural Networks, vol. 3, no. 5, 807-814, 1992.

[4] T. Takagi, and M. Sugeno, "Fuzzy identication of systems and its applications tomodelling and control, IEEE Transactions on Systems Man and Cybernetics",SMC-15 (1), 1985.

[5] K. Tanaka, and M. Sugeno, "Stability analysis and design of fuzzy control systems", Fuzzy Sets and Systems, vol. 45 no. 2, 135-156, 1992

[6] H. O. Wang, K. Tanaka, E. Griffin, "Parallel Distributed Compensation of Non-linear Systems by Takagi-Sugeno Fuzzy Model", Proceedings of 1995 IEEE International Conference on Fuzzy Systems, vol.2, pp.531-538, 1995.

[7] K. Tanaka, and H. O. Wang, "Fuzzy Control Systems Design and Analysis, A Linear Matrix Inequality Approach", John Wiley and Sons, 2001.
[8] S. K. Hong, and Y. Nam, "Stable fuzzy control system design with pole-placement constraint:An LMI approach", Computer in Industry, vol 51, pp. 1-11, 2003.

[9] M. Pokorny, and T. Docekal "Dynamic Tuning of the Optimal Fuzzy LQR Controller", Proc, IEEE SMC 2019, Bari, Italy, 2019.

[10] K. Tanaka, T. Ikeda, and H. O. Wang, "Robust stabilization of a class of uncertain non-linear systems via fuzzy control:Quadratic stability, $\mathrm{H}$ control theory, and linear matrix inequalities", IEEE Transactions on Fuzzy Systems, vol. 4 no. 1, pp. 1-13, 1996.

[11] R. G. Venkata, K. Padmanabhan, and S. Ananthi, "Optimal Control with Fuzzy State Space Modelling Using Riccati Equation", Int. Journal of Information and Electronic Engineering, vol. 2, no. 5, pp. 800-805, 2012.

[12] F. Khaber, K. Zehar, and A. Hamzaoui, "State Feedback Controller Design via Takagi-Sugeno Fuzzy Model:LMI Approach", Int. Journal of Mechanical and Mechatronics Engineering, vol. 2, no. 6 , pp. 836-841, 2008.

[13] A. Khajeh, and R. Ghazi, "GA-Based Optimal LQR Controller to Improve LVRT Capability of DFIG Wind Turbines", Iranian Journal of Electrical and Electronic Engineering, vol. 9, no. 3, pp, 167-176, 2013.

[14] J. G. G. Grijalva, "Control of a quadrotor using TS fuzzy techniques", PHD thesis, vol. University of Santa Catarina, no. Florianopolis, Equador, 2017.

[15] P. Gahinet et al , "The LMI Control Toolbox, Proc, of 33rd IEEE Conference on Decision and Control", Lake Buena Vista, USA, vol. 3, pp. 2038-2041, 1994.

[16] J. Löfberg, "YALMIP: A toolbox for modelling and optimization in MATLAB", IEEE International Conference on Robotics and Automation, New Orleans, USA, pp. 284-289, 2004.

[17] SEDUMI"Semidefinite programming solver", available com/SQLP/SeDuMi, 2019.

Received 28 December 2019

Miroslav Pokorný (Prof, Dr, Ing), born in 1941, graduated from the Brno University of Technology in 1963, where he passed his doctoral study from Technical Cybernetics and Informatics in 1994. Since 1964 he has worked in Research Institute of Metallurgy in Dobrá, since 1993 he has been with the Department of Cybernetics and Biomedical Engineering at the Faculty of Electrical Engineering and Informatics, VSB Technical University of Ostrava. He was appointed Associated Professor in 1995 and Full Professor in Technical Cybernetics in 2003. His primary interest includes the areas of artificial intelligence and process control.

Tomáš Dočekal (Ing), born in 1992, graduated from the VSB Technical University of Ostrava, Faculty of Electrical Engineering and Computer Science in 2016. At present he is pursuing his doctoral degree in Technical Cybernetics. He also cooperates on several scientific research projects. His field of research includes control and modelling of under-actuated non-linear systems with focus on inverted pendulums.

Danica Rosinová (Prof, Ing, PhD), born in 1961, graduated from the Slovak Technical University Bratislava, Faculty of Electrical Engineering in 1985 and obtained $\mathrm{PhD}$ degree in 1996. At present she is Professor with the Institute of Automotive Mechatronics, FEI STU Bratislava. Her eld of research includes robust stabilization of discrete time dynamic systems, control of large-scale systems, LMI approach in state-space robust control problems, optimization. She has published her scientific results at several IFAC conferences, in the International Journal of Control, Cybernetics and in other scientific journals in Slovakia and abroad. 